\title{
A Regulatory Role for the Insulin- and BDNF-Linked RORA in the Hippocampus: Implications for Alzheimer's Disease
}

\author{
George K. Acquaah-Mensah*, Nnenna Agu, Tayyiba Khan and Alice Gardner \\ Department of Pharmaceutical Sciences, School of Pharmacy, MCPHS University (Massachusetts College of \\ Pharmacy and Health Sciences), Worcester, Massachusetts, USA
}

Accepted 25 September 2014

\begin{abstract}
Alzheimer's disease (AD) is the leading cause of dementia. The etiology of AD remains, in large part, unresolved. In this study, gene expression (microarray) data from postmortem brains in normal aged as well as AD-affected brains in conjunction with transcriptional regulatory networks were explored for etiological insights. The focus was on the hippocampus, a brain region key to memory and learning. The transcriptional regulatory networks were inferred using a trees-based (random forests or extra-trees) as well as a mutual information-based algorithm applied to compendia of adult mouse whole brain and hippocampus microarray data. Network nodes representing human orthologs of the mouse networks were used in the subsequent analysis. Among the potential transcriptional regulators tied to insulin or brain-derived neurotrophic factor (INS1, INS2, BDNF), whose peptide products have been linked to AD, is the Retinoic Acid Receptor-Related Orphan Receptor (RORA). RORA is a nuclear receptor transcription factor whose expression is distinctly upregulated in the AD hippocampus. A notable cross-section of genes differentially expressed in the AD hippocampus was found to be linked to RORA in the networks. Furthermore, several genes associated with RORA in the networks, such as APP, DNMIL, and TIAl have been implicated in AD. Computationallyderived clusters and modules within the networks indicated strong ties between RORA and genes involved in the AD etiology. In addition, a functional mapping scheme using activity and interaction data affirmed the same network links to RORA. Thus, RORA emerges as a gene with a probable central role in the AD pathology/etiology.
\end{abstract}

Keywords: Alzheimer's disease, retinoids, RORA, transcriptional networks

\section{INTRODUCTION}

Alzheimer's disease $(\mathrm{AD})$ is a leading cause of dementia in the aging population [1]. Current numbers estimate 24 million individuals suffer from $\mathrm{AD}$ with an annual projected increase of 4.6 million underscoring the global health burden of the disease. The AD presentation is characterized by the progressive loss of

\footnotetext{
*Correspondence to: George K. Acquaah-Mensah, Department of Pharmaceutical Sciences, School of Pharmacy, MCPHS University (Massachusetts College of Pharmacy and Health Sciences), 19 Foster Street, Worcester, MA 01608, USA. Tel.: +1 508373 5643; Fax: +1 508890 5618; E-mail: george.acquaah-mensah@ mcphs.edu.
}

memory and cognition. The pathology of $\mathrm{AD}$ involves neuronal loss and the buildup of plaque, which contains extracellular deposits of amyloid- $\beta$, and neurofibrillary tangles made up of hyperphosphorylated tau [2-4]. In $\mathrm{AD}$, there are also cholinergic deficits in the basal forebrain cholinergic complex, a source of cholinergic projections to the cerebral cortex and the hippocampus [5]. The hippocampus is a particularly important region of the brain because of its role in learning and memory [6]. Neuronal loss in the hippocampus is associated with cognitive deficits in SAMP8 mice [7]. Furthermore, there are associations between changes in neurogenesis in the adult hippocampus, and $\mathrm{AD}$ pathogenesis [8]. 
Brain-derived neurotrophic factor (BDNF) is one of several growth factors important for memory formation and cell proliferation. Within the hippocampus, this neurotrophic factor is found in high levels, and the BDNF-trkB signaling system is sensitive to agerelated changes [9]. In a significant cross-section of AD-affected hippocampal pyramidal and basal forebrain neurons, cell cycle transition was aberrant resulting in the mitosis phase not being initiated and cells remaining tetraploid [10]. Endogenous BDNF is required for cell cycle regulation, as it is needed for inhibiting the $\mathrm{G}_{2} / \mathrm{M}$ transition in chick retinal ganglion cells [11]. In the postmortem AD hippocampus, BDNF mRNA expression is decreased [12]. Thus, it has been hypothesized that the decrease in BDNF-trkB expression in AD could facilitate the $\mathrm{G}_{2} / \mathrm{M}$ cell cycle transition and apoptosis in tetraploid neurons affected by $\mathrm{AD}[13]$.

Oxidative stress plays a very important role in $\mathrm{AD}$ pathology $[14,15]$. BDNF protects mouse hippocampal and cortical neurons against damage caused by hydrogen peroxide and $\mathrm{A} \beta$ fibrils [16]. BDNF inducers, such as lithium and $\mathrm{J} 147$, have been shown to mitigate symptoms of AD. For example, J147 prevents cognitive decline and facilitates memory in a transgenic rodent model of $\mathrm{AD}$ [17]. In this AD model, elevated levels of the inflammatory and oxidative stress biomarkers lipoxygenase, and heme oxygenase 1 , respectively, are significantly reduced in the presence of J147 [17].

Insulin is another peptide with strong links to AD [18]. Specifically, there are strong links between dysregulation of insulin function and $\mathrm{AD}$ [19]. Products of lipid peroxidation and glycoxidation are found in both $\mathrm{AD}$ and diabetes mellitus, a disease of which insulin dysfunction is a hallmark [20]. Thus, shared hallmarks between diabetes mellitus and AD include insulin resistance and reduced glucose metabolism [21]. Furthermore, both diseases are characterized by cognitive impairment [22], as well as shared pathways [23].

The aim of this study was to explore whole mouse brain and mouse hippocampus gene expression profiles and postmortem hippocampi of AD patients for further insights into transcriptional regulatory relationships between $B D N F$ and insulin and their influence on AD. Networks were generated on the basis of gene expression patterns of the mouse whole brain across a variety of microarray conditions representing genetic and pharmacologic perturbations. Mouse whole brain was used primarily because of a relative paucity of available brain region-specific mouse or human microarray data. Additionally, a smaller mouse hippocampus dataset was similarly used. Computational algorithms used included information theoretic and clustering algorithms. We identify RORA (Retinoic Acid ReceptorRelated Orphan Receptor), which has elevated expression in the AD hippocampus, as notable among the interaction partners of INS1, INS2, and BDNF. Sodhi and Singh have recently reviewed the neuroprotective role of retinoids and their link to AD [24]. Here, we hypothesize that RORA and several other gene products (itemized below) are key participants in molecular interaction events associated with AD.

\section{METHODS}

One hundred and sixty one human brain microarrays on the Affymetrix U133 Plus 2.0 Array ${ }^{\mathrm{TM}}$ platform from the Gene Expression Omnibus (GSE5281) were analyzed. The microarrays represent neuronal mRNA expression in 6 brain regions of AD-affected and control postmortem human brains. The brain regions are the entorhinal cortex, hippocampus, medial temporal gyrus, posterior cingulate, superior frontal gyrus, and primary visual cortex. Regarding the hippocampus, CA1 region pyramidal neurons were used, given that this region undergoes the most tangle formation in $\mathrm{AD}$ earliest [25]. There were 13 hippocampus samples (10 male; 3 female) not affected by $\mathrm{AD}$, and 10 affected by $\mathrm{AD}$ (6 male; 4 female). Background correction and data normalization were performed using the Robust Multi-array Average (RMA) procedure implementation in the affy package of Bioconductor $[26,27]$. Genes differentially expressed between the $\mathrm{AD}$ hippocampus and the normal hippocampus were identified using Significance Analysis of Microarrays (siggenes package) at a false discovery rate of $1 \%$ $[28,29]$. These data were subsequently superimposed on human orthologs of transcriptional regulatory networks reverse-engineered from mouse gene expression data: first, the mouse whole brain, and then the mouse hippocampus.

\section{Networks derived from whole mouse brain}

\section{Using ARACNe}

A human ortholog version of a previously reverse-engineered whole mouse brain transcriptional regulatory network [30] was created. The original network of genes involved in apoptosis, the response to oxidative stress, and inflammation was derived from 411 mouse whole brain microarray data from the Phenogen database [31], using the Algorithm for the Reconstruction of Accurate Cellular Networks [32] 
(ARACNe). Based on differential expression of genes in the postmortem AD hippocampus (described in the previous paragraph), active sub-networks were identified using default parameters of the jActive modules Cytoscape plug-in implementation [33, 34].

\section{Using GENIE 3}

A tree-based approach, Genie 3 [35], was subsequently used to infer a regulatory network on the basis of the gene expression data obtained from Phenogen [31] and used above. The underlying assumption is that the expression of any given gene under a given condition is a function of the expressions of other network genes. Thus, for each gene in a relevant compendium of normalized gene expression data, a learning sample is generated such that expression values of that gene serve as output, while the expression values of all other genes serve as input. For each such learning sample, a function is learned and a local ranking of all other genes (i.e., exception being the current gene) as potential regulators of the current gene is generated. The ranking is obtained by way of tree-based ensemble methods, Random Forests [36] and ExtraTrees [37]. Each learning sample is recursively split, using binary tests, each based on an input variable in an effort to reduce the variance of the output variable in the sub-samples generated. The bases for the local rankings are weights, calculated as sums of output variance reductions. An aggregate of all such local rankings is subsequently created. Thus, the top-scoring 1000 edges were selected to constitute a network for further analysis as discussed below. A human ortholog network was subsequently derived.

\section{Networks derived from mouse hippocampus}

The Gene Expression Omnibus datasets GSE32536 and GSE46871 represent hippocampal gene expression in transgenic mouse models of AD. In the GSE32536 (16-sample) dataset, the AD model mice used belonged to the B6;129-Psen1 ${ }^{\text {tm } 1 M p m}$ $\operatorname{Tg}$ (APPSwe,tauP301L)1Lfa/Mmjax strain [38]. In the case of the GSE46871 (6-sample) dataset, the AD model mice were of the strain B6;SJL$\operatorname{Tg}$ (APPSWE)2576Kha [39]. The raw data from these datasets were thus used to create a 22-sample compendium of mouse hippocampal gene expression data using the RMA procedure mentioned above. Probe sets included represent genes involved in apoptosis, the response to oxidative stress, and inflammation. Transcriptional regulatory networks were derived using both ARACNe and Genie 3, as described above.
Approaches for examining networks

\section{jActive modules}

The purpose of this algorithm is the identification of "active sub-networks", i.e., connected genes with unexpectedly high levels of differential expression [33]. There are primarily two steps in this algorithm:

1) Scoring of the amount of differential expression in a given sub-network: The level of significance of expression change for each sub-network gene is converted to a $\mathrm{z}$-score, and an aggregate $\mathrm{z}$ score for the entire sub-network computed. That aggregate z-score must exceed that of a background distribution (for a random set of genes, but independent of the network).

2) The identification of the highest scoring subnetworks, using a simulated annealing-based search algorithm with an additional heuristic to improve the efficiency of annealing. Ultimately all adjoining possibilities are explored and a local maximum obtained. The Greedy search algorithm may be used as an alternative. As a consequence, the resulting sub-network tends to be biologically meaningful.

The number of jActive modules was set at 5, the overlap threshold at 8; the Greedy Search method was used at a search depth of 1 and a maximum depth from start nodes of 2. High-scoring sub-networks, including genes associated with BDNF were identified.

\section{cis-Regulatory Elements}

Using the cis-Regulatory Elements in the Mammalian Genome (cREMaG) database [40], upstream regions of relevant sub-networks were examined for the presence of common cis-regulatory elements in genes of the active sub-networks.

\section{Functional mapper}

Furthermore, the Human Experimental/Functional Mapper (HEFalMp) [41], an aggregate functional mapping derived from close to 30 billion data points and over and 30,000 microarrays in more than 15,000 peerreviewed publications was explored for affirmation of functional relationships indicated by the data.

\section{$M C O D E$}

The Molecular Complex Detection (MCODE) algorithm [42], which detects significant clusters in complex networks, was applied along with the hippocampal $\mathrm{AD}$ differential expression data to the networks. MCODE identifies regions of high 
connectivity in large interaction networks, and consists primarily of the following steps:

1) Vertex weighting: measures the "cliquishness" of the neighborhood of each vertex. A clique, as used here, is a maximally connected graph. The density of the graph here is defined as the number of edges divided by the theoretical maximum number of edges possible. All vertices are assigned weights based on the densities of the local neighborhood. The central most densely connected sub-graph has the highest k-core of the graph (the k-core being the minimal degree of the graph), and the weight assigned a vertex is the product of a defined vertex core-clustering coefficient and the highest $\mathrm{k}$-core value of the local neighborhood.

2) Complex prediction: the vertex-weighted graph is the input for this stage. Beginning with the highest weighted vertex as seed, the complex is built by moving recursively from the seed vertex, and including only those vertices that have weights higher than a user-defined threshold (the vertex weight percentage parameter). If a vertex is included, its immediate neighbors are similarly assessed to determine if they are part of the complex. A vertex is assessed only once. The process ends when no more vertices can be added. The procedure is repeated for the next highest scoring vertex, and so on.

3) Post-processing: The alternatives for this optional stage are "fluff", "haircut", or both. Neighbors of vertices within the complex are added if they have not yet been seen, and their neighborhood density exceeds the user-defined fluff parameter (which is between 0 and 1). Under the haircut option, complexes are eliminated if they do not have a minimum degree of 2. If both are used, the fluff processing takes place first, and then the hair-cut option. The Degree cutoff used in these studies was 2 , and the Haircut cluster finding approach was used at a Node Score Cutoff of 0.2, a k-core of 2, and a maximum depth set at 100 .

Concurring predictions derived from regulatory networks generated using these distinct inference approaches (ARACNe and GENIE3) were deemed highly noteworthy and are incorporated into the discussion of current understanding of the AD etiology.

\section{RESULTS}

\section{Mouse whole brain}

\section{Network derived using ARACNe}

$B D N F$ was found to be directly associated with 23 other nodes by way of 44 edges in the ARACNegenerated network [30], which consisted of 1,256 nodes and 132,292 edges. (Repeat edges were connections between multiple probe sets representing the same genes). Of these nodes, RORA and BCLAF1 were expressed several fold more in the postmortem AD hippocampus (Table 1) than in the control postmortem AD hippocampus at very highly significant levels; FIS1, NRXN1, and HSPD1 had suppressed expression. RORA was further scrutinized, given its direct connection to 366 other nodes in this reverse-engineered whole brain transcriptional regulatory network, several of which were differentially expressed in the human AD hippocampus. Of the nodes linked to RORA, 39 had suppressed expression in the postmortem AD hippocampus (Table 2A), and 25 had elevated expression (Table 2B). Furthermore, RORA was connected to INSI via $G R M$, and to INS2 via $S P 1$.

In this network inferred using ARACNe [30], BDNF is in the same jActive module as RORA, APP, DNMIL, HSP90B1, CTNNB1, and NFE2L2. The RORA gene, whose expression is elevated in the AD hippocampus, was also directly linked to APP and $H S P 90 B 1$ both of which, like RORA, have elevated expression in this postmortem AD hippocampus dataset (Table 2B). (Regarding APP expression, it has to be said that other studies in other models have had different findings). RORA was similarly directly linked to DNMIL and $C T N N B 1$ both of which, in contrast to RORA, have suppressed expression in the AD hippocampus (Table 2A). Furthermore, $A P P$ whose association with $\mathrm{AD}$ was established and whose expression is elevated in the AD hippocampus is also directly associated both $D N M 1 L$ and $C T N N B 1$.

Table 1

Connectivity of $B D N F$-linked differentially expressed nodes in ARACNe-derived mouse whole brain network

\begin{tabular}{lccc}
\hline $\begin{array}{l}\text { Gene } \\
\text { symbol }\end{array}$ & $\begin{array}{c}\text { Number of } \\
\text { connections in } \\
\text { larger network }\end{array}$ & $\begin{array}{c}\text { Expression ratio } \\
\text { in hippocampus } \\
\text { AD-affected/control }\end{array}$ & $p$ value \\
\hline RORA & 1354 & 3.83 & $7.70 \mathrm{E}-005$ \\
BCLAF1 & 825 & 2.41 & $1.10 \mathrm{E}-006$ \\
HSPD1 & 94 & 0.49 & 0 \\
NRXN1 & 509 & 0.37 & 0 \\
FIS1 & 255 & 0.36 & $5.49 \mathrm{E}-006$ \\
\hline
\end{tabular}


Table 2A

RORA-linked genes with suppressed expression in the AD hippocampus (ARACNe-derived mouse whole brain network)

\begin{tabular}{|c|c|c|c|c|c|}
\hline $\begin{array}{l}\text { Gene } \\
\text { symbol }\end{array}$ & $\begin{array}{l}\text { Expression ratio } \\
\text { in hippocampus } \\
\text { AD-affected/control }\end{array}$ & $p$-value & $\begin{array}{l}\text { Gene } \\
\text { symbol }\end{array}$ & $\begin{array}{l}\text { Expression ratio } \\
\text { in hippocampus } \\
\text { AD-affected/control }\end{array}$ & $p$ value \\
\hline$Y W H A H$ & 0.09 & $1.83 \mathrm{E}-007$ & $D A P K 1$ & 0.37 & $5.85 \mathrm{E}-006$ \\
\hline$V D A C 1$ & 0.2 & $6.44 \mathrm{E}-005$ & $N R X N 1$ & 0.37 & 0 \\
\hline DHCR24 & 0.22 & 0 & PARK7 & 0.38 & $1.10 \mathrm{E}-006$ \\
\hline$Y W H A Z$ & 0.24 & $4.06 \mathrm{E}-005$ & HSPA9 & 0.39 & $1.83 \mathrm{E}-006$ \\
\hline PHB & 0.25 & $1.44 \mathrm{E}-005$ & BIRC6 & 0.4 & $1.39 \mathrm{E}-005$ \\
\hline$A P L P 2$ & 0.26 & 0 & $L T A 4 H$ & 0.4 & $1.35 \mathrm{E}-005$ \\
\hline NDUFA13 & 0.26 & 0 & OPA1 & 0.43 & $1.10 \mathrm{E}-006$ \\
\hline PRDX1 & 0.26 & $2.18 \mathrm{E}-005$ & FXR1 & 0.48 & $1.06 \mathrm{E}-005$ \\
\hline SMARCA4 & 0.27 & 7.32E-006 & CTNNB1 & 0.49 & $1.10 \mathrm{E}-006$ \\
\hline GLO1 & 0.28 & 7.50E-006 & SHROO M2 & 0.49 & $1.43 \mathrm{E}-005$ \\
\hline CUL3 & 0.29 & 4.44E-005 & $C S D E 1$ & 0.5 & $1.12 \mathrm{E}-005$ \\
\hline$P P P 2 R 1 A$ & 0.29 & 4.79E-005 & $M A D D$ & 0.51 & 0 \\
\hline SOD1 & 0.29 & $9.40 \mathrm{E}-005$ & SERINC3 & 0.51 & 7.32E-007 \\
\hline SYN2 & 0.29 & 0 & $F A F 1$ & 0.53 & $2.76 \mathrm{E}-005$ \\
\hline$D N M 1 L$ & 0.32 & $6.90 \mathrm{E}-005$ & NRCAM & 0.54 & $5.45 \mathrm{E}-005$ \\
\hline$T K T$ & 0.34 & $8.03 \mathrm{E}-005$ & ICAI & 0.58 & 7.32E-006 \\
\hline$S L C 23 A 2$ & 0.35 & $9.14 \mathrm{E}-007$ & PEX13 & 0.61 & $1.83 \mathrm{E}-007$ \\
\hline FIS1 & 0.36 & $5.49 \mathrm{E}-006$ & $P T K 2$ & 0.62 & $1.46 \mathrm{E}-006$ \\
\hline$P R K D C$ & 0.36 & $2.93 \mathrm{E}-006$ & SLC1A2 & 0.82 & 0 \\
\hline$A L S 2$ & 0.37 & 0 & & & \\
\hline
\end{tabular}

Table 2B

RORA-linked genes with elevated expression in the $\mathrm{AD}$ hippocampus (ARACNe-derived mouse whole brain network)

\begin{tabular}{|c|c|c|}
\hline $\begin{array}{l}\text { Gene } \\
\text { symbol }\end{array}$ & $\begin{array}{c}\text { Expression ratio } \\
\text { in hippocampus } \\
\text { AD-affected/control }\end{array}$ & $p$ value \\
\hline ERC1 & 4.45 & 0 \\
\hline HSP90B1 & 4.2 & 7.32E-007 \\
\hline PDCD6 & 3.79 & 7.92E-005 \\
\hline CFLAR & 3.64 & 4.63E-005 \\
\hline CADM1 & 3.46 & 5.49E-007 \\
\hline NRXN2 & 2.79 & 0 \\
\hline BAG4 & 2.69 & 0 \\
\hline RTN4 & 2.53 & $7.96 \mathrm{E}-005$ \\
\hline BCLAF1 & 2.41 & $1.10 \mathrm{E}-006$ \\
\hline MED1 & 2.36 & 0 \\
\hline GNAQ & 2.33 & $2.05 \mathrm{E}-005$ \\
\hline PIGT & 2.22 & 0 \\
\hline NFIB & 2.19 & $9.51 \mathrm{E}-006$ \\
\hline TIA1 & 2.1 & 4.10E-005 \\
\hline DAB1 & 1.97 & 2.67E-005 \\
\hline HMGB1 & 1.93 & 0 \\
\hline PBX1 & 1.85 & 4.37E-005 \\
\hline PLXNA2 & 1.82 & 4.39E-006 \\
\hline RABEP1 & 1.78 & $9.49 \mathrm{E}-005$ \\
\hline $\mathrm{SON}$ & 1.77 & 7.32E-006 \\
\hline GABRB1 & 1.71 & $8.25 \mathrm{E}-005$ \\
\hline RARG & 1.54 & 2.83E-005 \\
\hline MYCBP2 & 1.49 & 4.39E-006 \\
\hline APP & 1.47 & $2.52 \mathrm{E}-005$ \\
\hline PRKG1 & 1.37 & 4.21E-005 \\
\hline
\end{tabular}

cREMaG analysis showed that RORA has putative binding sites in the upstream regions of the following genes; YWHAH, HSPA9, PHB, CTNNB1, SYN2,
CSDE1, SLC23A2, MADD, NRXN1, NRCAM, RARG, GABRB1, PBX1, NF1B, NRXN2, and CADM1. These genes were not only directly linked to RORA based on this network (derived using ARACNE) but also importantly are differentially expressed in the AD hippocampus. This is a noteworthy observation and affirms the importance of RORA as a transcriptional regulator of note in $\mathrm{AD}$.

Functional Mapping (HEFalMp): Results of the HEFalMp runs also showed that RORA is functionally associated with the differentially expressed genes that were directly linked to RORA. RORA's functional relation with these genes is in the context of various biological processes. The following genes directly linked to RORA in the ARACNe-derived network and differentially expressed in the AD hippocampus had strong associations with RORA: PRKGl (0.89), GABRB1 (0.86), RARG (0.76), PLXNA2 (0.73), SYN2 (0.72), NRCAM (0.7), CADM1 (0.65), NRXN1 (0.59), SLC1A2 (0.58), ICA1 (0.56). The scores represent the extents of functional association the genes have (based of the published data as described [41]) with RORA. The scores range from 0 to 1 , with 1 being highly associated. Only scores above 0.5 are listed here. A longer list is available in Supplementary Table 2.

\section{Network derived using GENIE3}

The GENIE3-derived transcriptional regulatory network consisted of 539 nodes and 952 edges. The MCODE-generated clusters on this network showed 
Table 2C

RORA-linked genes with elevated expression in the AD hippocampus (ARACNe-derived mouse hippocampus network)

\begin{tabular}{|c|c|c|c|c|c|}
\hline $\begin{array}{l}\text { Gene } \\
\text { symbol }\end{array}$ & $\begin{array}{c}\text { Expression ratio } \\
\text { in hippocampus: } \\
\text { AD-affected/control }\end{array}$ & $p$ value & $\begin{array}{l}\text { Gene } \\
\text { symbol }\end{array}$ & $\begin{array}{c}\text { Expression ratio } \\
\text { in hippocampus: } \\
\text { AD-affected/control }\end{array}$ & $p$ value \\
\hline$C A D M 1$ & 3.4642 & $3.66 \mathrm{E}-007$ & $A P P$ & 1.4724 & $1.02 \mathrm{E}-005$ \\
\hline CHST11 & 3.25 & $3.66 \mathrm{E}-007$ & GRIN2B & 1.4537 & 0.002544 \\
\hline$R O R A$ & 3.0089 & 7.76E-004 & $C G A$ & 1.4411 & $2.60 \mathrm{E}-004$ \\
\hline HIPK2 & 2.9651 & $8.30 \mathrm{E}-004$ & MTF1 & 1.428 & 7.14E-004 \\
\hline CSRNP3 & 2.9077 & 0 & $M N T$ & 1.4089 & 0.003665 \\
\hline$N R 2 F 2$ & 2.7949 & $1.70 \mathrm{E}-004$ & CCR4 & 1.4066 & 7.10E-004 \\
\hline$B A G 4$ & 2.6914 & 0 & DNASE1 & 1.3967 & $9.51 \mathrm{E}-005$ \\
\hline$U B E 2 Z$ & 2.43 & 0 & CCR3 & 1.3961 & $2.19 \mathrm{E}-004$ \\
\hline$T C F 7 L 2$ & 2.3509 & $1.46 \mathrm{E}-006$ & $C D K N 2 A$ & 1.3753 & $6.53 \mathrm{E}-004$ \\
\hline$G A B R A 3$ & 2.3353 & $4.31 \mathrm{E}-004$ & $T B X 19$ & 1.3694 & 0.002116 \\
\hline$P P A R D$ & 2.2472 & $2.71 \mathrm{E}-004$ & PRF1 & 1.3506 & 0.002802 \\
\hline UNC $5 B$ & 2.2019 & $1.39 \mathrm{E}-005$ & $E 2 F 1$ & 1.3497 & 8.67E-004 \\
\hline$K R A S$ & 2.152 & $2.20 \mathrm{E}-004$ & NTN1 & 1.3389 & 0.002412 \\
\hline$P I K 3 R 1$ & 2.147 & $6.51 \mathrm{E}-004$ & $T B X 5$ & 1.3284 & 0.002845 \\
\hline TIA1 & 2.0959 & $1.66 \mathrm{E}-005$ & ZSCAN10 & 1.3246 & 0.002537 \\
\hline$F G F R 1$ & 2.0224 & $5.50 \mathrm{E}-004$ & CARD14 & 1.3055 & 0.002164 \\
\hline KCNMA 1 & 1.9243 & 0.002691 & $T G F B 2$ & 1.3013 & 0.00191 \\
\hline$P L X N A 2$ & 1.8239 & $1.46 \mathrm{E}-006$ & HOXD10 & 1.2878 & $3.91 \mathrm{E}-004$ \\
\hline SON & 1.771 & $2.56 \mathrm{E}-006$ & NCR1 & 1.2803 & $8.51 \mathrm{E}-004$ \\
\hline$B N I P 3 L$ & 1.7604 & 0.003694 & $F O X P 2$ & 1.2798 & 0.001942 \\
\hline$N U M B$ & 1.7574 & 0.001391 & NOS1 & 1.2628 & 7.17E-004 \\
\hline GHRHR & 1.7071 & $2.57 \mathrm{E}-004$ & CHRNA3 & 1.2603 & 0.001425 \\
\hline SIX3 & 1.592 & $5.40 \mathrm{E}-004$ & $A G E R$ & 1.2428 & 0.001689 \\
\hline TNFRSF14 & 1.5866 & $8.08 \mathrm{E}-004$ & $D N M 2$ & 1.2426 & 0.002566 \\
\hline$R A R G$ & 1.5411 & $1.10 \mathrm{E}-005$ & $D M B X 1$ & 1.1512 & 0.001743 \\
\hline CASP6 & 1.5228 & $2.03 \mathrm{E}-004$ & $B C L 2 L 14$ & 1.1444 & 0.001331 \\
\hline SOX 5 & 1.4832 & $8.30 \mathrm{E}-004$ & & & \\
\hline
\end{tabular}

the following: RORA, SMAD4, HIF1A, and CREB1 are all clustered together along with 18 other nodes in the highest scoring MCODE cluster of the GENIE3derived transcriptional regulatory network Fig. 1. As was seen in the jActive Module in the ARACNederived network, the upregulated RORA is directly linked to the down-regulated $O P A 1$ and $D N M 1 L$ in the AD hippocampus.

The five jActive modules of this network scored between 2.78 and 6.423 (Table 3). The four highest scoring modules each had PDCD6IP, PTK2, PLXNA2, RORA, NFIB, APP, CADM1, BIRC6, DAB1, RTN3, NRXN1, RABEP1, GNAQ, PBX1, OPA1, DHCR24, and $N R 2 F 1$ represented. As depicted in Fig. 2, most of the nodes in these modules are differentially expressed in the AD hippocampus.

\section{Mouse hippocampus}

The network reverse-engineered using ARACNe consisted of 1,204 nodes and 283,047 edges. The network reverse-engineered using Genie 3 consisted of 848 nodes and 1949 edges. As was done before, expression data from human postmortem brains (controls and
AD-affected) from GEO dataset GSE5281 was superimposed on the human ortholog of each network.

\section{Network derived using ARACNe}

The human ortholog version of the network derived executing ARACNe on the mouse hippocampus dataset originally consisted of, nodes and 283,047 edges. Of these 172,420 duplicated edges (resulting from multiple probe sets representing certain individual genes) were removed, leaving 110,627 unique edges. Among the most connected and differentially expressed nodes in this network were $N R X N 1, S O X 5$, NRXN3, RORA, RAC1, DNMIL, CAMK2A, MDH1, and $A P P$. RORA was directly connected to $S L C 23 A 2$, $N R X N 1, R A R G$, and $C A D M 1$, all listed as having RORA binding sites in their upstream regions using cREMaG. Also, RORA was directly linked to INSI, $I N S 2, A P P, D N M 1 L$, and PLXNA2. Fifty three of the genes directly tied to RORA had elevated expression (False discovery rate of 0.01 ); sixty nine had suppressed expression (Table 2C, D). Given the large number of regulatory links in this network, MCODEderived modules within it were examined for further insights. The highest-scoring module (score 196.35) 
Table 2D

RORA-linked genes with suppressed expression in the AD hippocampus (ARACNe-derived mouse hippocampus network)

\begin{tabular}{|c|c|c|c|c|c|}
\hline $\begin{array}{l}\text { Gene } \\
\text { symbol }\end{array}$ & $\begin{array}{c}\text { Expression ratio } \\
\text { in hippocampus: } \\
\text { AD-affected/control }\end{array}$ & $p$ value & $\begin{array}{l}\text { Gene } \\
\text { symbol }\end{array}$ & $\begin{array}{c}\text { Expression ratio } \\
\text { in hippocampus: } \\
\text { AD-affected/control }\end{array}$ & $p$ value \\
\hline SNAP25 & 0.1579 & 4.39E-005 & TACC1 & 0.606 & $2.41 \mathrm{E}-005$ \\
\hline DHCR24 & 0.2181 & $4.65 \mathrm{E}-005$ & $A T G 7$ & 0.6094 & $4.76 \mathrm{E}-005$ \\
\hline$Y W H A Z$ & 0.2417 & $1.66 \mathrm{E}-005$ & AIFM1 & 0.6153 & $6.12 \mathrm{E}-004$ \\
\hline$R A B E P 1$ & 0.3129 & 0.001656 & $P T K 2$ & 0.6238 & $9.14 \mathrm{E}-007$ \\
\hline$D N M 1 L$ & 0.3153 & $2.80 \mathrm{E}-005$ & $N F 1$ & 0.6344 & 0.004514 \\
\hline ELMO1 & 0.3164 & $2.56 \mathrm{E}-006$ & $P S M G 2$ & 0.6397 & 0.002142 \\
\hline$M I F$ & 0.3171 & $2.56 \mathrm{E}-006$ & ERCCl & 0.6472 & 0.003741 \\
\hline DNAJB6 & 0.3184 & $1.81 \mathrm{E}-005$ & PEX5 & 0.6476 & 0.002824 \\
\hline$M D H 1$ & 0.3237 & 0.002533 & $M A P K 8$ & 0.66 & $6.43 \mathrm{E}-004$ \\
\hline$B A G 5$ & 0.3345 & 0 & $M X 1$ & 0.6607 & 0.00226 \\
\hline$T K T$ & 0.3423 & $3.26 \mathrm{E}-005$ & ITGB1 & 0.667 & 0.003493 \\
\hline$S L C 23 A 2$ & 0.3489 & $3.66 \mathrm{E}-007$ & CHRNB2 & 0.6708 & $5.09 \mathrm{E}-004$ \\
\hline SNCA & 0.3521 & $9.54 \mathrm{E}-004$ & $D Y N L T 1$ & 0.6754 & 0.003352 \\
\hline$N R X N 3$ & 0.3523 & $1.00 \mathrm{E}-004$ & $S P 1$ & 0.6821 & $3.56 \mathrm{E}-004$ \\
\hline$A L S 2$ & 0.3654 & 7.06E-005 & TSC1 & 0.686 & 0.001604 \\
\hline$N R X N 1$ & 0.3694 & $8.93 \mathrm{E}-005$ & NEK6 & 0.6958 & 0.003818 \\
\hline HSP90B1 & 0.3954 & $3.06 \mathrm{E}-004$ & DNAJA3 & 0.697 & 4.69E-004 \\
\hline$L T A 4 H$ & 0.3984 & $5.12 \mathrm{E}-006$ & $B A R D 1$ & 0.7013 & 0.00177 \\
\hline$P R K C Z$ & 0.4113 & $9.14 \mathrm{E}-007$ & $C Y B B$ & 0.711 & 7.81E-004 \\
\hline$C A M K 1 D$ & 0.4193 & 0.001432 & POU $4 F 1$ & 0.7262 & $3.48 \mathrm{E}-004$ \\
\hline$M E D 1$ & 0.4288 & $1.76 \mathrm{E}-004$ & РТCH1 & 0.757 & 0.003627 \\
\hline PPT1 & 0.4362 & $1.12 \mathrm{E}-005$ & $S A A 1$ & 0.7808 & 0.003207 \\
\hline$T M 2 D 1$ & 0.4424 & $1.83 \mathrm{E}-004$ & TNFRSF4 & 0.7816 & 0.001278 \\
\hline$G S K 3 B$ & 0.4553 & $6.43 \mathrm{E}-004$ & $M D M 2$ & 0.783 & 0.002697 \\
\hline$A F G 3 L 2$ & 0.4632 & $1.08 \mathrm{E}-004$ & $A L B$ & 0.7843 & 0.004464 \\
\hline RHOA & 0.4712 & 0.001297 & SLC4A4 & 0.7912 & 0.002722 \\
\hline SYT1 & 0.4874 & 0.001157 & BIRC3 & 0.7947 & 0.001843 \\
\hline SERINC3 & 0.511 & $3.66 \mathrm{E}-007$ & $U B E 4 B$ & 0.8238 & 0.003841 \\
\hline$A P L P 2$ & 0.5445 & 0.001521 & NOX4 & 0.8322 & $8.16 \mathrm{E}-004$ \\
\hline DIDOI & 0.5527 & $2.77 \mathrm{E}-004$ & SLC6A4 & 0.8483 & 0.001825 \\
\hline$R A C 1$ & 0.5578 & 7.31E-004 & C9 & 0.8661 & 0.003474 \\
\hline PAFAH1B1 & 0.565 & 0.003763 & $C K A P 2$ & 0.8754 & 0.003874 \\
\hline PSEN2 & 0.57 & 0.004773 & PTPRC & 0.8765 & 0.002284 \\
\hline$C A M K 2 A$ & 0.5733 & $1.31 \mathrm{E}-004$ & $S L C 28 A 3$ & 0.8803 & $6.81 \mathrm{E}-004$ \\
\hline GABRB3 & 0.5756 & 0.001714 & & & \\
\hline
\end{tabular}

consisted of 444 nodes and 87,303 edges. Remarkably, all nodes in this module (including RORA) were directly linked to $C G A$, the gene for the alpha polypeptide associated with the glycoprotein hormones (chorionic gonadotropin, luteinizing hormone, follicle stimulating hormone, and thyroid stimulating hormone). Of note CGA had higher expression levels in the $\mathrm{AD}$-affected hippocampus.

\section{Network derived using GENIE3}

The 2000 highest-scoring edges computed between probe sets yielded a network of 1,949 edges between 848 gene symbol nodes. Among the most connected and differentially expressed nodes in this network include NFIB, RORA, STAT3, NFE2L2, BCLAF1, $C R E B B P$, and TCF7L2. RORA was present in each of the populated jActive modules, an indication of its connectedness to genes differentially expressed in $\mathrm{AD}$
(Supplementary Figs. 2 and 3). In this network, RORA was closely associated (linked directly, or by up to a few degrees) with several of the nodes found linked to it (above using cREMaG) and the ARACNe-derived whole mouse brain transcriptional regulatory network. Though not directly connected to $B D N F$ (and others highlighted above) in this network, RORA was directly connected to insulin signaling-related INSI, IGFI, and IGF1R (Supplementary Fig. 4). RORA was also connected to INS2 and to RARG by way of $N R 4 A 2$, and to $I G F 2$ by way of $M S X 1$.

$N R 4 A 2$ is a member of the steroid-thyroid hormoneretinoid receptor superfamily, and is down-regulated in the hippocampi of memory-impaired AD-modelmicein association with CREB-regulated transcription coactivator 1 activity [43] (Supplementary Fig.5). Suppressed expression of NR4A2, which is also linked directly to $R O R A$ and $R A R G$ in this network, is also associated 


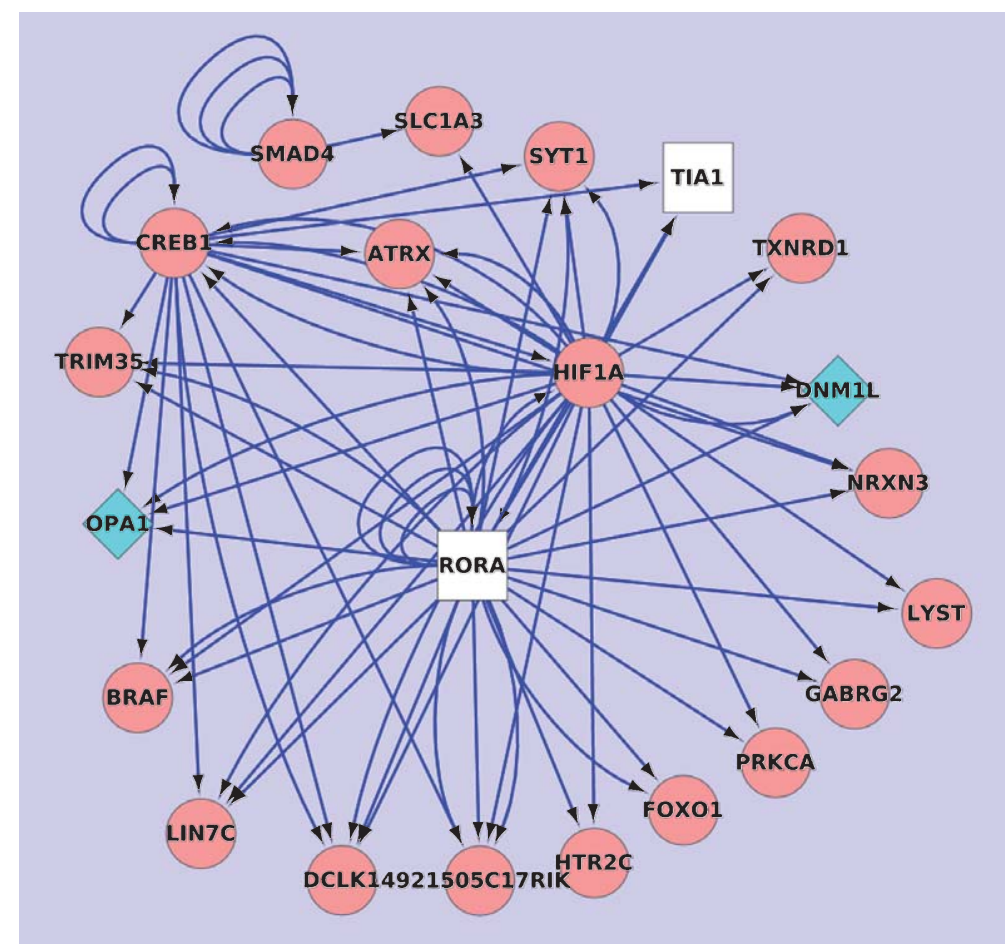

Fig. 1. RORA clusters with OPA1, TIA1, and DNM1L, along with other nodes. The network is derived via the GENIE3 algorithm from a compendium of 411 microarrays of the adult mouse whole brain, and the MCODE algorithm was used to generate this cluster. Diamond-shaped nodes represent genes whose expressions are suppressed in the human Alzheimer's disease (AD) hippocampus; rectangle-shaped nodes represent genes whose expressions are elevated in the AD hippocampus. OPA1, TIA1, DNMIL and other nodes are involved in processes relevant to the $\mathrm{AD}$ etiology. Multiple lines between any given pairs of nodes indicate the genes involved are represented by more than one probe set on the microarray and that the same relationship is detected when alternate probe sets are used.

Table 3

jActive modules in GENIE3-derived mouse whole brain network

\begin{tabular}{lcc}
\hline Rank & Size & Score \\
\hline 1 & 43 & 6.42 \\
2 & 63 & 6.33 \\
3 & 61 & 5.28 \\
4 & 47 & 2.96 \\
5 & 2 & 2.78 \\
\hline
\end{tabular}

with intracellular changes in dopaminergic neurons in Parkinson's disease and related diseases [44]. MSX1, like NR4A2, is linked with mid-brain dopaminergic neurons critical in Parkinson's disease [45].

\section{DISCUSSION}

Exploring beyond BDNF, a growth factor whose links to $\mathrm{AD}$ has been asserted [46, 47], genes whose expressions are statistically tied to $B D N F$ expression in transcriptional regulatory networks are prime candidates for further scrutiny (see Jimenez et al. [48]). Of the nodes linked to $B D N F$ in the ARACNe-derived whole mouse brain network (Supplementary Fig. 1), RORA stands out as being both very highly connected and upregulated in the AD hippocampus (Table 1). It is also remarkable that RORA is linked to INS1 as well as INS2 via the metabotropic glutamate receptor gene GRM2 in the same network. Further, RORA is directly linked to both $I G F 1$ and $I G F 1 R$, and is linked to $I G F 2$ via the glucose-regulated protein gene HSPA9. As has been pointed out above, there is insulin dysregulation associated with AD. RORA, also known as ROR1, ROR2, ROR3, RZRA, NR1F1, or RZR-ALPHA is a transcriptional factor that belongs to the super family of nuclear receptors. RORA is noted to be involved in autoimmune and metabolic disorders [49]. RORA is expressed in both neurons and glial cells and protects cerebellar neurons against oxidative stress [50]. RORA regulates and is responsive to sex-hormones, and has been linked with autism spectrum disorders [51, 52]. RORA emerges as an interesting node in the various networks in this report for a number of reasons. First, relative to the control hippocampus, the expression of the RORA gene is elevated in the AD hippocampus (Table 1). 


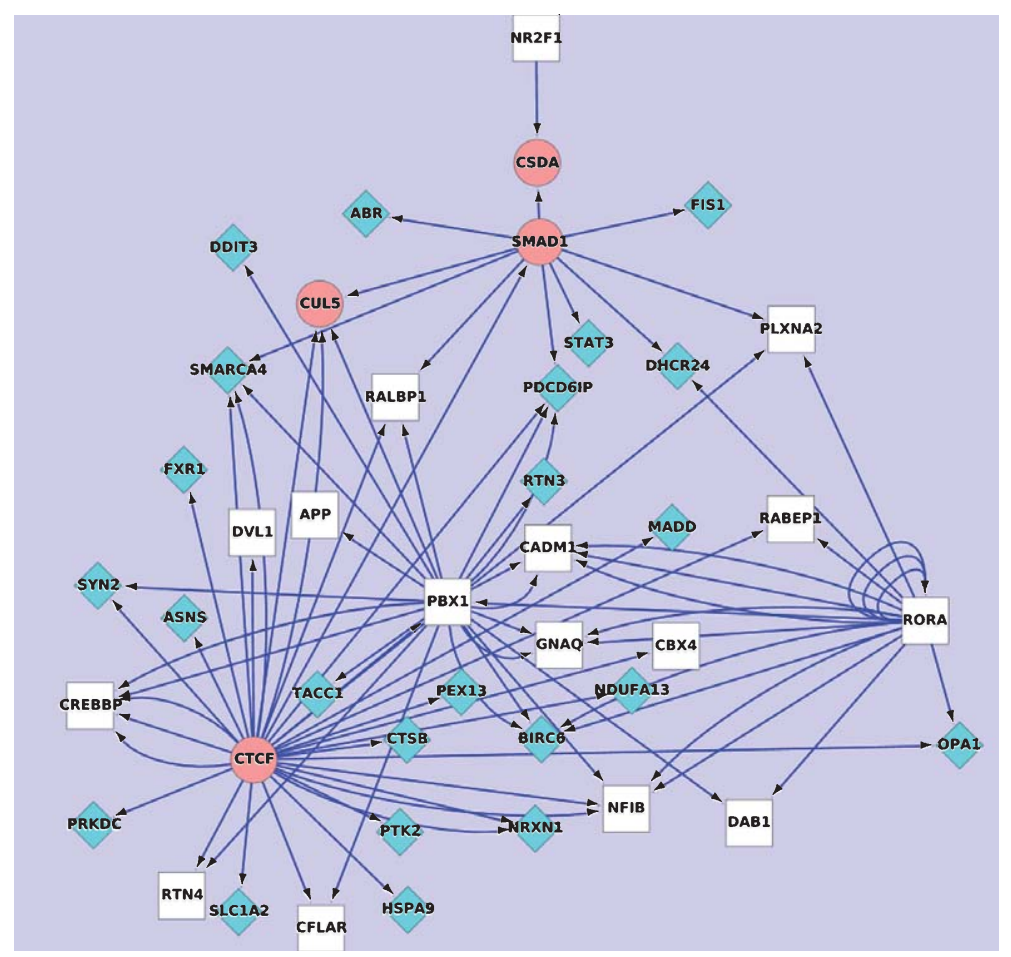

Fig. 2. RORA is highly connected in a network region of high occurrence of differentially expressed genes (jActive Module) in the human Alzheimer's disease (AD) hippocampus. A notable cross-section of the nodes representing differentially expresses nodes are either directly or indirectly linked to RORA in the network. The network is derived via the GENIE3 algorithm from a compendium of 411 microarrays of the adult mouse whole brain. Diamond-shaped nodes represent genes whose expressions are suppressed in the AD hippocampus; rectangle-shaped nodes represent genes whose expressions are elevated in the AD hippocampus. Multiple lines between any given pairs of nodes indicate the genes involved are represented by more than one probe set on the microarray and that the same relationship is detected when alternate probe sets are used.

Secondly, RORA is linked to retinoic acid receptor RARG and NR4A2; retinoids have been identified as potential therapeutic targets in late onset $\mathrm{AD}$ [53]. Genes linked to AD such as PS1, PS2, and BACE are regulated by retinoid signaling $[54,55]$.

Third, RORA is connected to several differentially expressed nodes (between the postmortem AD hippocampus and control) in the ARACNe-derived whole brain network (Table 2A, Table 2B) as well as the other networks. Further, as stated under the results section, the upstream regions of the differentially expressed YWHAH, HSPA9, PHB, CTNNB1, SYN2, CSDE1, SLC23A2, MADD, NRXN1, NRCAM, RARG, GABRB1, PBX1, NF1B, NRXN2, and CADM1 have putative binding sites for RORA.

Even though the human ortholog networks (derived based on expression profiles in the whole mouse brain or derived using expression profiles in the hippocampus only) are not identical, there are commonalities. For instance, directly linked to RORA in both ARACNe-derived networks (whole brain, or hippocampus only) are PTK2, APLP2, SERINC3,
LTA4H, NRXN1, ALS2, SLC23A2, TKT, DNM1L, $Y W H A Z$, and DHCR24, all of which have suppressed expressed in the human AD-affected hippocampus. Similarly, RORA-linked upregulated genes in both networks are APP, RARG, SON, PLXNA2, TIA1, BAG4, and $C A D M 1$.

In the whole brain network, and using the same postmortem $\mathrm{AD}$ hippocampus dataset, RORA occurs in the same jActive module as BDNF, DNMIL, and $A P P$, all of which have established associations with AD. In Supplementary Table 1, links of these RORA-associated genes to neurodegeneration/AD are summarized.

It is also noteworthy, that the ARACNe-derived networks and the GENIE3-derived networks indicate many of the same nodes as probable RORA targets. Furthermore, in terms of functional relationships to RORA, the HEFalMp scores listed in the results above (and in Supplementary Table 2) affirm the relationships of a cross-section of these nodes to RORA, even though this regularized Bayesian integration approach is orthogonal to either algorithm 
Table 4

Regulators and their targets in the best-scoring jActive module (GENIE3-derived mouse whole brain network)

\begin{tabular}{lll}
\hline & \multicolumn{2}{c}{ Predicted targets } \\
\cline { 2 - 3 } $\begin{array}{l}\text { Transcriptional } \\
\text { regulator(s) }\end{array}$ & \multicolumn{1}{c}{$\begin{array}{c}\text { Upregulated in } \\
\text { AD hippocampus }\end{array}$} & \multicolumn{1}{c}{$\begin{array}{c}\text { Down-regulated in } \\
\text { AD hippocampus }\end{array}$} \\
\hline RORA and PBX1 & PLXNA2, DAB1, NFIB, GNAQ, CADM1 & BIRC6 \\
RORA and CTCF & PBX1, RABEP1 & OPA1 \\
RORA and SMAD1 & - & DHCR24 \\
RORA and HIF1A & TIA1 & DNM1L \\
PBX1 & APP & DDIT3, RTN3, TACC1 \\
CTCF & CBX4, DVL1 & FXR1, ASNS, PRKDC, SLC1A2, CTSB, PTK2, \\
& & HSPA9, PEX13, NRXN1, NDUFA13, MADD \\
SMAD1 & - & ABR, STAT3, FIS1 \\
PBX1 and CTCF & CFLAR, RTN4, CREBBP & SYN2 \\
PBX1, CTCF and SMAD1 & - & SMARCA4, PDCD6IP \\
\hline
\end{tabular}

RORA and PBX1 are both upregulated in the AD hippocampus.

(ARACNe or GENIE3) [41]. The HEFalMp scores rely on literature findings and database entries and the weightings assigned, and so may be somewhat limited in characterizing undiscovered relationships. They, nonetheless, affirm in large measure the findings made via the other two approaches. Several of these network nodes are not discussed in-depth here. However, in the section following, nodes of $\mathrm{jActive}$ modules of the GENIE3-derived network are highlighted to illustrate the importance of RORA relationships in AD.

\section{jActive module components}

RORA is interesting also when one examines the four highest scoring jActive modules of the GENIE3derived whole brain transcriptional regulatory network (Table 3). These are connected nodes in the network with unexpectedly high levels of differential expression [33]. They all include PDCD6IP, PLXNA2, RORA, NFIB, APP, CADM1, BIRC6, DAB1, RTN3, NRXN1, RABEP1, GNAQ, PBX1, OPA1, DHCR24, and NR2F1. Based on the data and inferences, these are all regulatory targets of RORA, PBX1, CTCF, SMAD1, and/or HIF1A (Table 4, Fig. 2). Several of these genes are also found in the top-scoring jActive modules from the GENIE3-derived hippocampus only network (Supplementary Figs. 2 and 3). These genes associated with RORA are interesting not only because they are mostly differentially expressed in the AD hippocampus, but also because of the processes they are involved in (Supplementary Table 1). Furthermore, when the postmortem AD hippocampus dataset (GSE5281) is examined along gender lines, RORA has increased expression in both males and females (FDR 5\%); also in males as well as females, genes associated with the pathway "RORA activates circadian gene expression" (in REACTOME [56]) occur more in the list of upregulated genes than one would expect by chance (Supplementary Table 3).

Thus, overall indications from the data presented in this report are that RORA is linked in important ways to molecules that could be playing important roles in the AD etiology. This makes RORA an important gene/gene product in the etiology/pathology of AD. Indeed recent ChIP-on-chip studies have indicated an over-representation of genes linked with learning, memory and cognition among probable regulatory targets of RORA [57]. Thus, on the basis of the data presented here, we here posit an important link between hippocampal RORA and AD.

\section{DISCLOSURE STATEMENT}

Authors' disclosures available online (http://www.jalz.com/disclosures/view.php?id=2570 ).

\section{SUPPLEMENTARY MATERIAL}

The supplementary material is available in the electronic version of this article: http://dx.doi.org/ 10.3233/JAD-141731.

\section{REFERENCES}

[1] Evans D, Funkenstein H, Albert M, Scherr P, Cook N, Chown M, Hebert L, Hennekens C, Taylor J (1989) Prevalence of Alzheimer's disease in a community population of older persons. Higher than previously reported. JAMA 262, 2551-2556.

[2] Moossy J, Zubenko GS, Martinez AJ, Rao GR (1988) Bilateral symmetry of morphologic lesions in Alzheimer's disease. Arch Neurol 45, 251-254.

[3] Delacourte A, David JP, Sergeant N, Buee L, Wattez A, Vermersch P, Ghozali F, Fallet-Bianco C, Pasquier F, Lebert F, Petit H, Di Menza C (1999) The biochemical pathway of neurofibrillary degeneration in aging and Alzheimer's disease. Neurology 52, 1158-1165. 
[4] Braak H, Braak E, Bohl J, Reintjes R (1996) Age, neurofibrillary changes, A beta-amyloid and the onset of Alzheimer's disease. Neurosci Lett 210, 87-90.

[5] Schliebs R, Arendt T (2011) The cholinergic system in aging and neuronal degeneration. Behav Brain Res 221, 555-563.

[6] Squire LR (1992) Memory and the hippocampus: A synthesis from findings with rats, monkeys, and humans. Psychol Rev 99, 195-231.

[7] Li G, Cheng H, Zhang X, Shang X, Xie H, Zhang X, Yu J, Han $\mathrm{J}$ (2013) Hippocampal neuron loss is correlated with cognitive deficits in SAMP8 mice. Neurol Sci 34, 963-969.

[8] Mu Y, Gage FH (2011) Adult hippocampal neurogenesis and its role in Alzheimer's disease. Mol Neurodegener 6, 85.

[9] Halbach OB (2010) Involvement of BDNF in age-dependent alterations in the hippocampus. Front Aging Neurosci 2, 36.

[10] Yang Y, Geldmacher DS, Herrup K (2001) DNA replication precedes neuronal cell death in Alzheimer's disease. J Neurosci 21, 2661-2668.

[11] Morillo SM, Escoll P, de la Hera A, Frade JM (2010) Somatic tetraploidy in specific chick retinal ganglion cells induced by nerve growth factor. Proc Natl Acad Sci U S A 107 , 109-114.

[12] Murray KD, Gall CM, Jones EG, Isackson PJ (1994) Differential regulation of brain-derived neurotrophic factor and type II calcium/calmodulin-dependent protein kinase messenger RNA expression in Alzheimer's disease. Neuroscience 60 , 37-48.

[13] Frade JM, Lopez-Sanchez N (2010) A novel hypothesis for Alzheimer disease based on neuronal tetraploidy induced by p75 (NTR). Cell Cycle 9, 1934-1941.

[14] Bonda DJ, Wang X, Perry G, Nunomura A, Tabaton M, Zhu $X$, Smith MA (2010) Oxidative stress in Alzheimer disease: A possibility for prevention. Neuropharmacology 59, 290-294.

[15] Dumont M, Wille E, Stack C, Calingasan NY, Beal MF, Lin MT (2009) Reduction of oxidative stress, amyloid deposition, and memory deficit by manganese superoxide dismutase overexpression in a transgenic mouse model of Alzheimer's disease. FASEB J 23, 2459-2466.

[16] Ill-Raga G, Ramos-Fernandez E, Guix FX, Tajes M, BoschMorato M, Palomer E, Godoy J, Belmar S, Cerpa W, Simpkins JW, Inestrosa NC, Munoz FJ (2010) Amyloid-beta peptide fibrils induce nitro-oxidative stress in neuronal cells. J Alzheimers Dis 22, 641-652.

[17] Chen Q, Prior M, Dargusch R, Roberts A, Riek R, Eichmann C, Chiruta C, Akaishi T, Abe K, Maher P, Schubert D (2011) A novel neurotrophic drug for cognitive enhancement and Alzheimer's disease. PLoS One 6, e27865.

[18] De Felice FG, Lourenco MV, Ferreira ST (2014) How does brain insulin resistance develop in Alzheimer's disease? Alzheimers Dement 10, S26-S32.

[19] El Khoury N, Gratuze M, Papon M, Bretteville A, Planel E (2014) Insulin dysfunction and tau pathology. Front Cell Neurosci 8, 22

[20] Reddy VP, Zhu X, Perry G, Smith MA (2009) Oxidative stress in diabetes and Alzheimer's disease. J Alzheimers Dis 16 763-774.

[21] Zhao W, Townsend M (2009) Insulin resistance and amyloidogenesis as common molecular foundation for type 2 diabetes and Alzheimer's disease. Biochim Biophys Acta 1792, 482-496.

[22] Kodl CT, Seaquist ER (2008) Cognitive dysfunction and diabetes mellitus. Endocr Rev 29, 494-511.

[23] Sims-Robinson C, Kim B, Rosko A, Feldman EL (2010) How does diabetes accelerate Alzheimer disease pathology? Nat Rev Neurol 6, 551-559.
[24] Sodhi RK, Singh N (2014) Retinoids as potential targets for Alzheimer's disease. Pharmacol Biochem Behav 120, 117123.

[25] Liang WS, Dunckley T, Beach TG, Grover A, Mastroeni D, Walker DG, Caselli RJ, Kukull WA, McKeel D, Morris JC, Hulette C, Schmechel D, Alexander GE, Reiman EM, Rogers J, Stephan DA (2007) Gene expression profiles in anatomically and functionally distinct regions of the normal aged human brain. Physiol Genomics 28, 311-322.

[26] Irizarry RA, Hobbs B, Collin F, Beazer-Barclay YD, Antonellis KJ, Scherf U, Speed TP (2003) Exploration, normalization, and summaries of high density oligonucleotide array probe level data. Biostatistics 4, 249-264.

[27] Irizarry R, Gautier L, Cope L (2003) An R package for analyses of Affymetrix oligonucleotide arrays. In The Analysis of Gene Expression Data: Methods and Software (Statistics for Biology and Health), Parmigiani G, Garett ES, Irizarry RA, Zeger SL, eds. Springer Science+Business Media, New York, pp. 102-119.

[28] Schwender H, Ickstadt K, Krause A (2003) Assessing the false discovery rate in a statistical analysis of gene expression data. 49th Biometric Conference of the German Region of the International Biometric Society, Wuppertal, Germany.

[29] Benjamini Y, Hochberg Y (1995) Controlling the false discovery rate: A practical and powerful approach to multiple testing. J Roy Statist Soc Ser B (Methodological) 57, 289-300.

[30] Acquaah-Mensah GK, Taylor RC, Bhave SV (2012) PACAP interactions in the mouse brain: Implications for behavioral and other disorders. Gene 491, 224-231.

[31] Bhave SV, Hornbaker C, Phang TL, Saba L, Lapadat R, Kechris K, Gaydos J, McGoldrick D, Dolbey A, Leach S, Soriano B, Ellington A, Ellington E, Jones K, Mangion J, Belknap JK, Williams RW, Hunter LE, Hoffman PL, Tabakoff B (2007) The PhenoGen informatics website: Tools for analyses of complex traits. BMC Genet 8,59 .

[32] Margolin AA, Nemenman I, Basso K, Wiggins C, Stolovitzky G, Dalla Favera R, Califano A (2006) ARACNE: An algorithm for the reconstruction of gene regulatory networks in a mammalian cellular context. BMC Bioinformatics 7 (Suppl 1), S7.

[33] Ideker T, Ozier O, Schwikowski B, Siegel AF (2002) Discovering regulatory and signalling circuits in molecular interaction networks. Bioinformatics 18 (Suppl 1), S233-40.

[34] Shannon P, Markiel A, Ozier O, Baliga NS, Wang JT, Ramage D, Amin N, Schwikowski B, Ideker T (2003) Cytoscape: A software environment for integrated models of biomolecular interaction networks. Genome Res 13, 2498-2504.

[35] Huynh-Thu VA, Irrthum A, Wehenkel L, Geurts P (2010) Inferring regulatory networks from expression data using treebased methods. PLoS One 5, e12776.

[36] Breiman L (2001) Random forests. Mach Learning 45, 5-32.

[37] Geurts P, Ernst D, Wehenkel L (2006) Extremely randomized trees. Mach Learning 63, 3-42.

[38] Oddo S, Caccamo A, Shepherd JD, Murphy MP, Golde TE, Kayed R, Metherate R, Mattson MP, Akbari Y, LaFerla FM (2003) Triple-transgenic model of Alzheimer's disease with plaques and tangles: Intracellular $A \beta$ and synaptic dysfunction. Neuron 39, 409-421.

[39] Hsiao K, Chapman P, Nilsen S, Eckman C, Harigaya Y, Younkin S, Yang F, Cole G (1996) Correlative memory deficits, Abeta elevation, and amyloid plaques in transgenic mice. Science 274, 99-102.

[40] Piechota M, Korostynski M, Przewlocki R (2010) Identification of cis-regulatory elements in the mammalian genome: The cREMaG database. PLoS One 5, e12465. 
[41] Huttenhower C, Haley EM, Hibbs MA, Dumeaux V, Barrett DR, Coller HA, Troyanskaya OG (2009) Exploring the human genome with functional maps. Genome Res 19, 1093-1106.

[42] Bader GD, Hogue CW (2003) An automated method for finding molecular complexes in large protein interaction networks. BMC Bioinformatics 4, 2.

[43] Saura CA (2012) CREB-regulated transcription coactivator 1-dependent transcription in Alzheimer's disease mice. $\mathrm{Neu}$ rodegener Dis 10, 250-252.

[44] Chu Y, Le W, Kompoliti K, Jankovic J, Mufson EJ, Kordower JH (2006) Nurr1 in Parkinson's disease and related disorders. J Comp Neurol 494, 495-514.

[45] Joksimovic M, Yun BA, Kittappa R, Anderegg AM, Chang WW, Taketo MM, McKay RD, Awatramani RB (2009) Wnt antagonism of Shh facilitates midbrain floor plate neurogenesis. Nat Neurosci 12, 125-131.

[46] Zhang F, Kang Z, Li W, Xiao Z, Zhou X (2012) Roles of brain-derived neurotrophic factor/tropomyosin-related kinase B (BDNF/TrkB) signalling in Alzheimer's disease. J Clin Neurosci 19, 946-949.

[47] Boiocchi C, Maggioli E, Zorzetto M, Sinforiani E, Cereda C, Ricevuti G, Cuccia M (2013) Brain-derived neurotrophic factor gene variants and Alzheimer disease: An association study in an Alzheimer disease Italian population. Rejuvenation Res 16, 57-66.

[48] Jimenez S, Torres M, Vizuete M, Sanchez-Varo R, SanchezMejias E, Trujillo-Estrada L, Carmona-Cuenca I, Caballero C, Ruano D, Gutierrez A, Vitorica J (2011) Age-dependent accumulation of soluble amyloid beta (Abeta) oligomers reverses the neuroprotective effect of soluble amyloid precursor protein-alpha (sAPP(alpha)) by modulating phosphatidylinositol 3-kinase (PI3K)/Akt-GSK-3beta pathway in Alzheimer mouse model. J Biol Chem 286, 18414-18425.

[49] Solt LA, Burris TP (2012) Action of RORs and their ligands in (patho)physiology. Trends Endocrinol Metab 23, 619-627.
[50] Boukhtouche F, Doulazmi M, Frederic F, Dusart I, Brugg B, Mariani J (2006) RORalpha, a pivotal nuclear receptor for Purkinje neuron survival and differentiation: From development to ageing. Cerebellum 5, 97-104.

[51] Sarachana T, Xu M, Wu RC, Hu VW (2011) Sex hormones in autism: Androgens and estrogens differentially and reciprocally regulate RORA, a novel candidate gene for autism. PLoS One 6, e17116.

[52] Nguyen A, Rauch TA, Pfeifer GP, Hu VW (2010) Global methylation profiling of lymphoblastoid cell lines reveals epigenetic contributions to autism spectrum disorders and a novel autism candidate gene, RORA, whose protein product is reduced in autistic brain. FASEB $J \mathbf{2 4}, 3036-3051$.

[53] Goodman AB (2006) Retinoid receptors, transporters, and metabolizers as therapeutic targets in late onset Alzheimer disease. J Cell Physiol 209, 598-603.

[54] Obulesu M, Dowlathabad MR, Bramhachari P (2011) Carotenoids and Alzheimer's disease: An insight into therapeutic role of retinoids in animal models. Neurochem Int 59, 535-541.

[55] Lee H, Casadesus G, Zhu X, Lee H, Perry G, Smith MA Gustaw-Rothenberg K, Lerner A (2009) All-trans retinoic acid as a novel therapeutic strategy for Alzheimer's disease. Expert Rev Neurotherapeutics 9, 1615-1621.

[56] Croft D, Mundo AF, Haw R, Milacic M, Weiser J, Wu G, Caudy M, Garapati P, Gillespie M, Kamdar MR, Jassal B, Jupe S, Matthews L, May B, Palatnik S, Rothfels K, Shamovsky V, Song H, Williams M, Birney E, Hermjakob H, Stein L, D'Eustachio P (2014) The Reactome pathway knowledgebase. Nucleic Acids Res 42, D472-7.

[57] Sarachana T, Hu VW (2013) Genome-wide identification of transcriptional targets of RORA reveals direct regulation of multiple genes associated with autism spectrum disorder. $\mathrm{Mol}$ Autism 4, 14-2392-4-14. 